\title{
Structural Regulation of Lanthanum Oxychloride and its Enhanced Photosynthetic Activity
}

\author{
Xie Liyan \\ Putian University, Fujian, Putian, 351100
}

Keywords: Structural Regulation, Lanthanum Oxychloride

\begin{abstract}
BiOCl has high photocatalytic efficiency due to its unique laminar structure, reasonable restraining zone and stable property, which has attracted a lot of researches from domestic and foreign scholars. However, it has the disadvantage of narrow absorption band, so it needs to be modified by metal, semiconductor and carbon materials. The difficulty in the study of bismuth chloride is the stability and load of bismuth chloride composite.
\end{abstract}

\section{Introduction}

At present, the energy crisis and environmental pollution have gradually affected the survival and development of human beings and become a worldwide problem. At present, photocatalytic technology can provide green hydrogen energy through solar energy decomposition of water, and also decompose organic pollutants in sewage, providing new support for energy and environmental protection. The traditional semiconductor photocatalysts are mainly $\mathrm{TiO} 2$ and CdS, etc. The disadvantage of these catalysts is that they have large bandgap width, which makes the catalytic efficiency low ${ }^{[1-2]}$.Therefore, we need to develop a catalyst with high photoefficiency and high catalytic activity.

In recent years, the two-dimensional material in terms of energy, catalytic show a broad application prospect, especially layered bismuth chloride, the material is non-toxic, stable chemical properties, optical properties of stable structure, easy to adjust and moderate band structure characteristic, in the light, showed excellent physical and chemical properties.

\section{Yttrium Oxychloride Material}

$\mathrm{BiOCl}$ has high anisotropy, and its crystal structure is $\mathrm{PbFCl}$, belonging to tetragonal system. Bismuth chloride is a V-VI-VII combinational semiconductor, which is formed into a layered structure along the (001) direction under the action of van der Waals force ${ }^{[3]}$. Because of bismuth oxide layer formed between the oxygen atoms and bismuth atoms within the covalent bond and covalent bond formed between chlorine and bismuth atoms exist difference, which makes bismuth chloride in photocatalytic decomposition of water and wastewater treatment has broad application prospects. Bismuth oxychloride has high catalytic activity is mainly due to: (1) after the valence electrons gain energy excitation to the conduction band in orbit, will be in the process of the electronic - hole, this feature provides more space for polarized atoms, at the same time accelerate the separation of electronic - hole, so that to improve the efficiency of photocatalytic ${ }^{[4]}$.(2) $\mathrm{BiOCl}$ belongs to the indirect transition zone semiconductor, so the excited electrons need to pass a certain $\mathrm{K}$ layer to reach the valence band, which reduces the probability of the excited electrons and holes combination. Because BiOCl has unique structure and indirect transition mode, photoelectron and hole can be separated effectively, which improves photocatalytic efficiency.

\section{Structural Regulation and Composite Modification of Bismuth Chloride}

\subsection{Structural regulation of bismuth chloride}

The structure regulation of bismuth chloride was mainly controlled by $\mathrm{PH}$ value control and surfactant addition. Literature [6] analyzed the existing problems of complex process, high energy 
consumption and easy introduction of other impurities in the current structural regulation of bismuth chlorooxide, and proposed a new structural regulation process of bismuth chlorooxide. The process flow is shown in figure 1

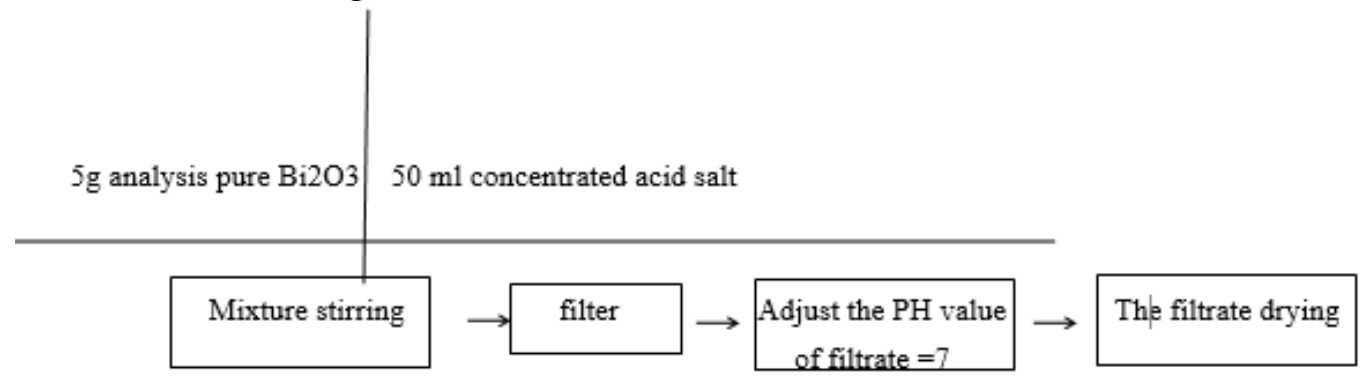

Fig. 1 flow chart of the new structure control process of bismuth chloride

The results of XRD diffraction analysis showed that the prepared bismuth chloride had high crystallinity and large grain size.Literature[3] studied the regulation method of $\mathrm{Bi3O} 4 \mathrm{Cl}$ on highly exposed crystal surface of $\{001\}$.In this method, 91\%\{001\}Bi3O4C nanoparticles were prepared by a pot of water thermal synthesis method. Through XRD, SEM and other technologies, it was found that $\mathrm{Bi} 3 \mathrm{O} 4 \mathrm{Cl}\{001\}$ crystal surface could induce stronger internal electric field, thus improving photocatalytic efficiency.

\subsection{Composite modification of bismuth chloride}

Although bismuth chloride has high photocatalytic efficiency, it has some defects. For example, the absorption band of a single photocatalyst is narrow and the structure is unstable. Therefore, the photocatalytic performance of bismuth oxychloride needs to be improved, so its performance needs to be modified.At present, bismuth chloride has been modified by metal, semiconductor and carbon materials.

\subsubsection{Metal - bismuth chloride composite modification}

The method is tantamount to dope the surface of yttrium oxychloride. Metal ions can provide photo-generated electrons, reduce the recombination of holes and electrons, and increase photosynthetic efficiency. Literature [7] used normothermia method to dope Ti and BiOCl together to prepare a $\mathrm{BiOCl} / \mathrm{BiOI}$ catalyst with higher catalytic efficiency and improve the efficiency of lanthanum oxychloride.The Literature [6] BiOCl1-xBrx was prepared using in-situ ion exchange technology, and prepared photocatalyst has higher catalytic efficiency. Literature [8-9] uses Sn, Mn, etc. to modify the yttrium oxychloride. The results show that adding the above metal elements can improve the photosynthetic efficiency ofyttrium oxychloride.

\subsubsection{Semiconductor - yttrium oxychloride composite modification}

In order to improve the electron mobility, the photocatalytic efficiency can be improved by combining the semiconductor with bismuth chloride. Literature [10] using solvent hot method producing ultra-thin BiOCL nano film, using high resolution projection electorate in electron microscopy, electron diffraction analysis of the exposed on the surface of BiOCL (001) crystal plane, analyzes its performance results show that the photocatalytic efficiency increased significantly. Literature[11] used reverse microemulsion method to prepare BiOCl/TiO2, among which $\mathrm{nBiOCl} / \mathrm{nTiO}=0.75$ time catalytic efficiency was the highest.2.2.3 Carbon Materials yttrium oxychloride composite modification

Carbon materials have excellent conductivity, and their application to yttrium oxychloride reduces the recombination rate of electron holes. In addition, the surface area of carbon materials is high, which can increase the contact area between reactants and improve the photosynthetic efficiency. Literature [12] uses colloquial method to prepare carbon-containing yttrium oxychloride material, and analyzes its microstructure and morphology through X-ray diffractometer and a transmission electron microscope. Finally, it is found that adding carbon material can make carbon atom and yttrium oxychloride contact. The photosensitive carrier separation improves the 
photosynthetic efficiency. Literature [6] explored the intrinsic nanophase carbon doping mechanism. The addition of carbon atoms can replace the lattice chlorine atoms and improve the photosynthetic efficiency.

\section{Conclusion}

Other composite modification methods include non-metallic doping, biox-bioy $(\mathrm{X}, \mathrm{Y}=\mathrm{Cl}, \mathrm{Br}, \mathrm{I})$ system, and modification of bismuth chloride into a magnetic catalyst.

Bismuth oxychloride in photocatalysis has a good application prospect, at present the research is still in its infancy, there are a lot of problems unresolved, such as: bismuth oxychloride composite stability and load, bismuth oxychloride and complex interface between properties.

\section{References}

[1] Honda K. Photolysis of water at a semiconductor electrode [J]. Nature, 1972, 238.

[2] Ochiai T,Masuko K,Tago S,et al.Synergistic water-treatment reactors using a tio2-modified ti-mesh filter[J]. Water, 2013, 5(3):1101-1115.

[3] LiJie. Structure regulation and photocatalytic activity enhancement of bismuth chloride layer [D]. Central China Normal University, 2016.

[4] Wu Feifei, Ma Chunyang, Jiao Jinlong, et al. Preparation and pyrolysis of bismuth chloride nanoparticles [J]. Journal of silicate, 2016, 44(7):948-952.

[5] Zhang Xiaoqiong.Preparation, structure and properties of $\mathrm{BiOCl}$ nanofunctional thin film materials [D]. Sun Yat-sen University, 2010.

[6] Jiang Yi.Controlled preparation and modification of selected BiOCl nanoparticles on $\{001$ surface [D]. Chongqing University, 2016.

[7] Wang R, Jiang G, Wang X, et al. Efficient visible-light-induced photocatalytic activity over the novel Ti-doped BiOBr microspheres[J]. Powder Technology, 2012, 228(3):258-263.

[8] Tu X, Qian S, Chen L, et al. The influence of Sn(II) doping on the photoinduced charge and photocatalytic properties of $\mathrm{BiOBr}$ microspheres[J].Journal of Materials Science, 2015, 50(12):4312-4323.

[9] Sarwan B. Influence of Mn2+ Ion on the Surface of BiOCl Catalyst for Photocatalytic Degradation of Methylene Green under Visible Light Illumination[C]// Materials Science Forum. 2013:194-205.

[10] Guan Meil. Controlled preparation and photocatalytic properties of bismuth oxide semiconductor nanomaterials with 2-d ultra-thin halide [D].China University of Science and Technology, 2014.

[11] Zhang L,Xu W, Fang J, et al. Decoration of BiOI quantum size nanoparticles with reduced graphene oxide in enhanced visible-light-driven photocatalytic studies[J]. Applied Surface Science, 2012, 259(41):441-447.

[12] Sun J, Song J, Gondal M A, et al. Preparation of g-C 3 N 4 /BiOX (X = Cl, Br, I) composites, and their photocatalytic activity under visible light irradiation[J]. Research on Chemical Intermediates, 2015, 41(10):6941-6955. 